\title{
A NOTE ON SUMMABILITY METHODS AND SPECTRAL ANALYSIS
}

BY

CARL S. HERZ1

We are concerned with analyzing the spectrum of a bounded measurable function on the real line by means of certain summability methods. If $f \in L^{1}$, the Fourier transform is $\hat{f}(t) \equiv \int \exp (-i t x) f(x) d x$ and $Z(f)$ denotes the set of zeros of $\hat{f}$. Given $\phi \in L^{\infty}$ we may form the convolution $f \circ \phi(x) \equiv \int f(y) \phi(x-y) d y$. The spectrum of $\phi$ is defined by $\Lambda(\phi)=\cap Z(f)$ where the intersection is taken over all $f \in L^{1}$ such that $f \circ \phi \equiv 0$.

The underlying heuristic principle of this investigation is that $t \notin \Lambda(\phi)$ if and only if the trigonometric integral $\int \exp (-i s x) \phi(x) d x$ is summable, in some suitable sense, to 0 in a neighborhood of $t$. Since we do not assume that $\int_{x}^{x+1}|\phi(y)| d y=o(1)$ as $|x| \rightarrow \infty$, ordinary convergence is not suitable. Beurling $\left(^{2}\right)$ has treated Abel summability and Pollard, [4], the $(R, 2)$ method. However their results may be extended to a large class of summability methods which are quite easy to describe. (Some of our theorems are new even for the Abel and $(R, 2)$ cases.)

Definition. A function $k \in L^{1}$ is a "spectral kernel" if $k(0)=1$ and $k(x)$ $=\int_{|x|}^{\infty} k^{\prime}(y) d y$ where $k^{\prime} \in L^{1}$. (We shall consider $k^{\prime}$ extended to negative arguments as an odd function.)

Set $\Phi_{h}(t)=\int \exp (-i t x) k(h x) \phi(x) d x$. Taking the limit as $h \rightarrow 0$ gives a regular summability method.

THEOREM 1. If $k$ is a spectral kernel, $\Phi_{h}(t) \rightarrow 0$ uniformly in any closed set at positive distance from $\Lambda(\phi)$. More precisely, if $t$ is at a distance $\delta$ from $\Lambda(\phi)$, $\left|\Phi_{h}(t)\right| \leqq \delta^{-1} v\left(\delta h^{-1}\right)\|\phi\|_{\infty}$ where $v$ depends only on $k$ and $v(\tau) \rightarrow 0$ as $\tau \rightarrow \infty$.

Proof. It suffices to establish the inequality for $t=0$. We assume the open interval $(-\delta, \delta)$ does not meet $\Lambda(\phi)$. From this it follows, as we shall see in a moment, that if $g \in L^{1}$ and $\hat{g}(t)=h^{-1} \hat{k}\left(h^{-1} t\right)$ in $|t| \geqq \delta$ then $\Phi_{h}(0)$ $\equiv \int k(h x) \phi(x) d x=\int g(x) \phi(x) d x$. Given $f \in L^{1}$, let $V_{\tau}(\hat{f})=\inf \|g\|_{1}$, where $\hat{g}(t)$ $=\hat{f}(t)$ in $|t| \geqq \tau$. Clearly $\left|\Phi_{h}(0)\right| \leqq V_{\delta}\left\{h^{-1} \hat{k}\left(h^{-1} t\right)\right\}$. $\|\phi\|_{\infty}$; however $V_{\delta}\left\{h^{-1} \hat{k}\left(h^{-1} t\right)\right\}=h^{-1} V_{\delta h^{-1}}(\hat{k})=h^{-1} V_{\delta h^{-1}}\left\{(-i t)^{-1} \hat{k}^{\prime}\right\}$. By Sz-Nagy's generalization of Bohr's inequality, [5], $V_{\tau}\left\{(-i t)^{-1} \hat{f}\right\} \leqq(\pi / 2 \tau) V_{\tau}(\hat{f})$ for any $f \in L^{1}$. Hence $V_{\delta}\left\{h^{-1} \hat{k}\left(h^{-1} t\right)\right\} \leqq(\pi / 2 \delta) V_{\delta h^{-1}}\left(\hat{k}^{\prime}\right)$. Also for $f \in L^{1}, V_{\tau}(\hat{f}) \rightarrow 0$ as $\tau \rightarrow \infty$.

Received by the editors August 16, 1956.

(1) The research for this paper was supported by the United States Air Force under Contract No. AF-18(600)-685 monitored by the Office of Scientific Research.

(2) Beurling's results are scattered in several papers and in lecture notes. The most relevant of these is $[2]$ where the essential ideas are given. 
The statement of the theorem has $v(\tau)=(\pi / 2) V_{\tau}\left(\hat{k}^{\prime}\right)$. To see the equality used at the beginning of the proof observe that if $\lambda>1$, the function $\phi(-\lambda x)$ has its spectrum interior to the set $|t| \geqq \delta$. Since the Fourier transform of $f(x)=k(h x)-g(x)$ vanishes on this set, i.e., on a neighborhood of the spectrum of $\phi(-\lambda x)$, it follows from a corollary of Wiener's Tauberian Theorem, see for example [3], that $\int f(x) \phi(\lambda x) d x=0$. Letting $\lambda$ tend to 1 from above we have the desired equality.

For $t \in \Lambda(\phi),\left|\Phi_{h}(t)\right| \leqq h^{-1}\|k\|_{1}\|\phi\|_{\infty}$. Neither this estimate nor the one of Theorem 1 can be improved as can be seen by taking $\phi(x) \equiv 1$. However, these estimates are misleading as far as the average behavior is concerned. Parseval's relation yields

$$
\left\{(2 \pi)^{-1} \int\left|\Phi_{h}(t)\right|^{2} d t\right\}^{1 / 2} \leqq h^{-1 / 2}\|k\|_{2}\|\phi\|_{\infty} .
$$

The corresponding analogue of Theorem 1 is the simpler result.

THEOREM 2. Let $k$ be a spectral kernel and $\Lambda_{\dot{0}}$ the set of points at distance $\geqq \delta$ from $\Lambda(\phi)$. Then $\left\{(2 \pi)^{-1} \int_{\Lambda_{\delta}}\left|\Phi_{h}(t)\right|^{2} d t\right\}^{1 / 2} \leqq \delta^{-1 / 2} w\left(\delta h^{-1}\right)\|\phi\|_{\infty}$ where w depends only on $k$ and $w(\tau) \rightarrow 0$ as $\tau \rightarrow \infty$.

Proof. The reasoning is similar to that of Theorem 1. Suppose $g \in L^{1}$ and $\hat{g}(t)=h^{-1} \hat{k}\left(h^{-1} t\right)$ in $|t| \geqq \delta$. Then if $s \in \Lambda_{\delta}, \Phi_{h}(s)=\int \exp (-i s x) g(x) \phi(x) d x$, so $(2 \pi)^{-1} \int_{\Lambda_{\delta}}\left|\Phi_{h}(t)\right|^{2} d t \leqq \int|g(x) \phi(x)|^{2} d x \leqq|| \phi \|_{\infty}^{2} \int|g(x)|^{2} d x$. The infimum of $\int|g(x)|^{2} d x$ taken over the set specified above is simply $(2 \pi)^{-1} \int_{|t| \geq \delta}\left|h^{-1} \hat{k}\left(h^{-1} t\right)\right|^{2} d t$ $=(2 \pi h)^{-1} \int|t| \geq \delta h^{-1}|\hat{k}(t)|^{2} d t$. Set $\quad w^{2}(\tau)=\tau \cdot(2 \pi)^{-1} \int_{|t| \geq \tau}|\hat{k}(t)|^{2} d t$. Then $\left\{(2 \pi)^{-1} \int_{\Lambda_{\delta}}\left|\Phi_{h}(t)\right|^{2} d t\right\}^{1 / 2} \leqq \delta^{-1 / 2} w\left(\delta h^{-1}\right)\|\phi\|_{\infty}$, and since $\hat{k}(t)=o\left(t^{-1}\right)$ as $t \rightarrow \infty$, $w(\tau)=o(1)$ as $\tau \rightarrow \infty$.

Suppose $f \in L^{1}, \phi \in L^{\infty}$ and we form the convolution $\psi=f \circ \phi$. There arises the question of the equi-convergence of $\hat{f}(t) \Phi_{h}(t)$ and $\Psi_{h}(t)$ $=\int \exp (-i t x) k(h x) \psi(x) d x$. A statement equivalent to the first sentence of Theorem 1 is that if $T$ is a closed set and $\hat{f}$ is constant on an $\epsilon$-neighborhood of $T$ then $\Psi_{h}$ and $\hat{f} \Phi_{h}$ are uniformly equi-convergent in $T$. More interesting is the fact that if $(1+|x|) f(x) \in L^{1}$ and $\lim _{y \rightarrow \infty}(2 y)^{-1} \int_{-y}^{y}|\phi(x)| d x=0$ then $\Psi_{h}$ and $f \Phi_{h}$ are uniformly equi-convergent everywhere. However, no matter how rapidly $|f|$ decreases at $\infty, \Psi_{h}$ and $f \Phi_{h}$ need not be equi-convergent everywhere. In particular, let $\phi(x)=i \operatorname{sgn} x$ and let $f$ be arbitrary except that $(1+|x|) f(x) \in L^{1}$. Then $\lim _{h \rightarrow 0} \Psi_{h}(0)-\hat{f}(0) \Phi_{h}(0)=\lim _{h \rightarrow 0} \Psi_{h}(0)=2(d \hat{f} / d t)(0)$, regardless of what spectral kernel is used. Actually this example is completely typical of the type of exceptional behavior which can occur. For $\phi \in L^{\infty}$ let $\Lambda_{0}(\phi)$ be the set of points $t$ at which

$$
\lim _{j \rightarrow \infty} y^{-1} \int_{0}^{y}\{\exp (-i t x) \phi(x)-\exp (i t x) \phi(-x)\} d x \neq 0 .
$$

It is known that every point of $\Lambda_{0}(\phi)$ is a cluster point of $\Lambda(\phi)$ and that $\Lambda_{0}(\phi)$ 
has measure zero $\left({ }^{3}\right)$.

THEOREM 3. Suppose $k$ is a spectral kernel, $\phi \in L^{\infty}, \int(1+|x|)|f(x)| d x<\infty$, and $\psi=f \circ \phi$. Then $\Psi_{h}-\hat{f} \Phi_{h}$ is uniformly bounded and $\Psi_{h}(t)-\hat{f}(t) \Phi_{h}(t) \rightarrow 0$ as $h \rightarrow 0$ for all $t$ with the exception of the null set where both $t \in \Lambda_{0}(\phi)$ and $d f / d t \neq 0$.

Proof. What we actually prove is that $\Psi_{h}(t)-\hat{f}(t) \Phi_{h}(t)=-i(d \hat{f} / d t)$. $F_{h}(t)+o(1)$ where $F_{h}(t)=h \int_{0}^{\infty} k^{\prime}(h x)\{\exp (-i t x) \phi(x)-\exp (i t x) \phi(-x)\} d x$ and $o$ depends only on $k^{\prime}, f$, and $\|\phi\|_{\infty}$. By Wiener's Tauberian theorem, $\lim _{h \rightarrow 0} F_{h}(t)$ $=\lim _{y \rightarrow \infty} y^{-1} \int_{0}^{y}\{\exp (-i t x) \phi(x)-\exp (i t x) \phi(-x)\} d x$ whenever the limit on the right exists; so the theorem follows directly. For $y \neq 0$, set

$$
F_{h}(t ; y)=y^{-1} \int \exp (-i t x)\{k(h x)-k[h(x+y)]\} \phi(x) d x .
$$

An easy computation shows that

$$
\Psi_{h}(t)-\hat{f}(t) \Phi_{h}(t)=-\int F_{h}(t ; y) \exp (-i t y) y f(y) d y .
$$

To conclude the proof we claim $F_{h}(t ; y)-F_{h}(t) \rightarrow 0$ boundedly as $h \rightarrow 0$. The calculation is effected by substituting for $k$ in terms of $k^{\prime}$ in the definition of $F_{h}(t ; y)$, whence

$$
\begin{aligned}
\left|F_{h}(t ; y)-F_{h}(t)\right| & =\left|\int\left\{y^{-1} \int_{h x}^{h x+h y} k^{\prime}(u) d u-h k^{\prime}(h x)\right\} \exp (-i t x) \phi(x) d x\right| \\
& \leqq\|\phi\|_{\infty} \int(h y)^{-1} \int_{0}^{h y}\left|k^{\prime}(x+u)-k^{\prime}(x)\right| d u d x \\
& =\|\phi\|_{\infty}(h y)^{-1} \int_{0}^{h y} \int\left|k^{\prime}(x+u)-k^{\prime}(x)\right| d x d u \\
& \leqq 2\left\|k^{\prime}\right\|_{1}\|\phi\|_{\infty}
\end{aligned}
$$

and $\rightarrow 0$ as $h \rightarrow 0$ for each $y$.

Equi-convergence in the mean is a much simpler matter. A trivial generalization of a result of Beurling, [1], is

THEOREM 4. Suppose $k$ is a spectral kernel, $\phi \in L^{\infty}, \int\left(1+|x|^{1 / 2}\right)|f(x)| d x$ $<\infty$, and $\psi=f \circ \phi$. Then $\int\left|\Psi_{h}(t)-\hat{f}(t) \Phi_{h}(t)\right|^{2} d t \rightarrow 0$ as $h \rightarrow 0$.

Next we turn to some questions in the converse direction from Theorems

(3) The latter statement follows from an affirmative answer given by W. H. J. Fuchs and others to the author's Research Problem (Bull. Amer. Math. Soc. Research Problem 62-1-2). The solution is unpublished, but the idea is simple. One observes that it is sufficient to take a sequence of $y^{\prime}$ s, say $y=n^{2}$, in the limit. Then by Plancherel's theorem one has a sequence of functions converging rapidly in the mean to zero. Such a sequence must also converge almost everywhere to zero. 
1 and 2. An elementary result using few of the assumptions on $k$ is this one.

Theorem 5. If for some $\epsilon>0, \lim \inf \int_{t-e}^{t+e}\left|\Phi_{h}(s)\right| d s=0$, then $t \notin \Lambda(\phi)$.

Proof. Choose $f \in L^{1}$ such that $\hat{f}(t) \neq 0$ and $\hat{f}(s)=0$ for $|s-t| \geqq \epsilon$.

$$
(2 \pi)^{-1} \int \exp (i s x) \Phi_{h}(s) \hat{f}(s) d s=\int k(h y) \phi(y) f(x-y) d y .
$$

Since $k(h y) \rightarrow 1$ boundedly as $h \rightarrow 0$, the right hand side converges to $f \circ \phi$. Hence the left hand side converges, and our assumption ensures that the limit is zero. Thus $f \circ \phi=0$; by definition $\Lambda(\phi) \subset Z(f)$ so $t \notin \Lambda(\phi)$.

The succeeding theorem involves a hypothesis about uniqueness whose verification may be a difficult and delicate matter.

Definition. The summability kernel $k$ is of type $U L^{\infty}$ if $\phi \in L^{\infty}$ and $\Phi_{h}(t) \rightarrow 0$ everywhere imply $\phi=0$ almost everywhere.

Theorem 6. Suppose $k$ is a spectral kernel of type $U L^{\infty}$ and $\phi \in L^{\infty}$. If $\Phi_{h}(s) \rightarrow 0$ in a neighborhood of the point $t$ then $t \notin \Lambda(\phi)$.

Proof. Suppose $\Phi_{h}(s) \rightarrow 0$ in $[t-\epsilon, t+\epsilon]$ for some $\epsilon>0$. By Baire's category theorem, given a sequence $h_{n} \rightarrow 0$, there exist points $t_{1}, t_{2}, t_{3}, t_{4}$ with $t-\epsilon<t_{1}$ $<t_{2}<t<t_{3}<t_{4}<t+\epsilon$ such that $\Phi_{h_{n}}(s) \rightarrow 0$ boundedly for $s \in\left[t_{1}, t_{2}\right]$ and $s \in\left[t_{3}, t_{4}\right]$. According to Theorem 5 the intervals $\left(t_{1}, t_{2}\right)$ and $\left(t_{3}, t_{4}\right)$ are complementary to $\Lambda(\phi)$. Now choose $f$ such that $\int(1+|x|)|f(x)| d x<\infty, \hat{f}(s)=0$ when $s \leqq t_{1}$ or $s \geqq t_{4}$, and $\hat{f}(s)=1$ for $t_{2} \leqq s \leqq t_{3}$. Put $\psi=f \circ \phi$. The derivative of $\hat{f}$ is 0 on $\Lambda(\phi)$ so by Theorem $3, \Psi_{h}(s) \rightarrow 0$ everywhere. Since $k$ is of type $U L^{\infty}$, this implies $\psi \equiv 0$, i.e., $t \notin \Lambda(\phi)$.

Combining Theorems 1 and 6 we have

COROLlaRY. Under the hypotheses of Theorem 6, if $\Phi_{h}(t)>0$ in an open interval, the convergence is uniform in each closed subinterval.

For a discussion of ordinary convergence, $k(x)=1$ for $|x| \leqq 1,=0$ for $|x|>1$, see [6]. In this case modifications of all the preceding theorems are valid under the hypothesis $\int_{x}^{x+1}|\phi(y)| d y=o(1)$ as $|x| \rightarrow \infty$. More generally, under some appropriate assumption of the form " $\phi$ is asymptotically small," e.g., $\phi(x)=o(1)$ or $\phi \in L^{a}, q<\infty$, one can replace the requirement that $k$ be a spectral kernel by the conditions: $k \in L^{1} \cap L^{\infty}, k(0)=1, k$ is continuous at 0 , and $\int|k(x+y)-k(x)| d x=O(y)$ as $y \rightarrow 0$. Then Theorem 3 holds in the form: $\Psi_{h}(t)-\hat{f}(t) \Phi_{h}(t) \rightarrow 0$ uniformly, while the proof of Theorem 4 is unmolested. Less precise versions of Theorems 1 and 2 may be derived from Theorems 3 and 4 respectively.

\section{BIBLIOGRAPHY}

1. Arne Beurling, Sur la composition d'une fonction sommable et d'une fonction borrnée, C.R. Acad. Sci. Paris vol. 225 (1947) pp. 274-275. 
2. - Sur une classe de fonctions presque périodiques, loc. cit. pp. 326-328.

3. Roger Godemont, Théorèmes Tauberiens et theorie spectrale, Ann. Ecole. Norm. vol. 64 (1947) pp. 119-138.

4. Harry Pollard, The harmonic analysis of bounded functions, Duke Math. J. vol. 20 (1953) pp. 499-512.

5. Bela Sz.-Nagy, Über gewisse Extremalfragen bei transformierte trigonometrische Entwicklungen, Berichte über die Verhandlungen der Sächsischen Akademie der Wissenschaften zu Leipzig. Mathematisch-Physische Klasse vol. 90 (1938) pp. 103-134 and vol. 91 (1939) pp. $3-24$.

6. Antoni Zygmund, Sur la théorie riemannienne des séries trigonométriques, Math. Zeit. vol. 24 (1926) pp. 47-104.

CORNELl UNIVERSity, ITHACA, N. Y. 